disorder have not been investigated, despite its potential relevance in this disorder, given its high cardiovascular and metabolic comorbidities and the encouraged maintenance of social rhythms in its management. This study aimed to explore the effectiveness of an adjunctive walking program in the acute treatment of bipolar disorder.

Methods: The sample consisted of a retrospective cohort of in-patients at a private psychiatric hospital with a primary diagnosis of bipolar disorder, who were admitted from January 2004 to December 2005. All patients were invited to participate in a 40-min walking group that took place on weekdays. Those who reliably attended the walking group (participants) were compared against those who never attended (nonparticipants), using the Clinical Global Impression (CGI) scales and the 21-item Depression Anxiety Stress Scales (DASS).

Results: The participants $(n=24)$ and nonparticipants $(n=74)$ were comparable in age, length of stay, bipolar subtype distribution, and baseline CGI and DASS measures, except for a lower DASS stress subscore for the participants (19.4 vs. 25.3, $P=0.049$ ). The groups did not differ in their discharge CGI scores, but participants showed significantly lower scores on DASS (23 vs. 44.6, $P=0.005)$ and all its subscales (depression 7.2 vs. $13.7, P=0.048$; anxiety 6.6 vs. $13.8, P=0.002$; stress 9.2 vs. $17.1, P=0.01)$ at the time of discharge.

Conclusions: Physical activity may have an adjunctive therapeutic role in bipolar disorder. Further investigation with randomized controlled trials is warranted.

\section{The utility of the Clinical Global Impression Scale in the clinical setting}

\section{F Ng ${ }^{1,2}$, T Trauer $^{2}$, M Bernardo ${ }^{2}$, S Campbell ${ }^{3}$, T Callaly', S Dodd'², M Berk ${ }^{2}$}

'Barwon Health; ${ }^{2}$ The University of Melbourne, Melbourne, Australia; and ${ }^{3}$ Healthscope, Melbourne, Australia

Background: The Clinical Global Impression (CGI) scale is an established outcome measure in psychopharmacology research and has been applied to specific disorders, including schizophrenia, anxiety disorders, depression and bipolar disorder. Its simplicity and ability to transcend diagnostic boundaries support its utility in the general clinical setting. This study was conducted to test the validity of the CGI in a private psychiatric in-patient setting.

Methods: Consecutive admissions $(n=786)$ to a private psychiatric hospital from January 2004 to December 2005 were studied. Retrospective data were collected on four outcome measures that were routinely administered at admission and discharge. These were the self-rated 21-item Depression Anxiety Stress Scales (DASS-21) and the Mental Health Questionnaire (MHQ-14), and the clinicianrated CGI and Health of the Nation Outcome Scales (HoNOS). In relation to the CGI, only the severity (CGI-S) and global improvement (CGI-I) subscales were used. Comparative statistical analyses were performed.

Results: The numbers of completed CGI ratings were 624 admission CGI-S, 614 discharge CGI-S and 610 CGI-I. The admission and discharge CGI-S scores were correlated $(r=0.40)$, and the indirect improvement measures obtained from their differences were highly correlated with the direct CGI-I scores $(r=0.71)$. The CGI-S categories reflected similar trends in scores on the other three measures, and the CGI-I showed parallel changes with improvement on HoNOS.

Conclusions: The CGI-S and CGI-I are comparable to other measures of illness severity and improvement. They appear to be valid instruments in the private psychiatric in-patient setting.

\section{Alzheimer's disease, delusions and cognitive decline}

\section{0'Connor ${ }^{1,2}$, S Rossell'}

'Mental Health Research Institute; and ${ }^{2}$ The University of Melbourne, Melbourne, Australia

Background: The authors reviewed studies published between 1992 and 2005 that reported on the prevalence and phenomenology of delusions in Alzheimer's disease (AD), as well as any relationship with cognitive decline.

Methods: The terms 'delusions, cognitive and Alzheimer's disease/dementia' were used to search the PubMed and PsychINFO databases. Empirical investigations and reviews were included in our report but were dependent upon quantitative data on the above factors being available.

Results: Data from a meta-analysis show that the overall prevalence for delusions in $\mathrm{AD}$ is $36 \%$. There is, however, a broad range of reported prevalence rates across studies, from $9 \%$ to $70 \%$. Variations in prevalence rates are because of methodological differences, such as inconsistent consideration of neuroleptic use, participants being included at various stages of $A D$ and failure to consider other neuropsychiatric symptoms. One study did address factors that lead to inconsistent findings and subsequently reported that $34 \%$ of a sample of patients with $\mathrm{AD}$ were found to experience delusions and that these patients were at a higher risk of 
functional and cognitive decline. The phenomenology of $\mathrm{AD}$ delusions has not been adequately addressed; neither has whether AD delusions relate to specific cognitive deficits.

Conclusions: Delusions in AD are common and are related to greater rates of cognitive decline. Future studies that explore the relationship between $\mathrm{AD}$, delusions and cognitive decline need to control for methodological issues and need to examine the phenomenology of delusions and their relationship with specific cognitive deficits.

\section{Are affective prosodic deficits evident in bipolar disorder?}

\section{A O'Regan, SL Rossell, NR Joshua}

Mental Health Research Institute of Victoria, Melbourne, Australia

Background: Deficits in affective prosodic processing have been well established in patients with schizophrenia. Yet, no study has examined this skill in a bipolar sample, this was the aim of the current study.

Methods: Three matched groups (patients with bipolar disorder, patients with schizophrenia and healthy controls) completed four affective prosody tasks from the Comprehensive Affective Testing System (CATS); emotional prosody discrimination (EPD), name emotional prosody (NEP), attend to prosody during conflicting prosody (CPP) and attend to meaning during conflicting prosody (CPM).

Results: No significant group effects were found for any of the four tasks on either correct reaction time (RT) or accuracy measures. A task effect, however, was found across all four tasks showing a similar pattern for the RT (correct) and accuracy data; superior performance on EPD, then CPP, followed by NEP then CPM.

Conclusions: The lack of group differences for each of the four tasks may be a reflection of the CATS paradigms lacking the capacity to effectively discriminate subtle differences in performance. An extensive literature indicates that individuals with schizophrenia and bipolar disorder do tend to perform worse on a range of affect tasks that use fearful and sad stimuli. Therefore, the lack of significant group effects in the current tasks may be because of an insufficient number of stimuli per affective category used in each task, thus poor power; alternatively, incongruent accent must be considered, that is, CATS uses American not Australian actors. Further study is recommended using experimental measures of affective prosody.

\section{Omega-3 fatty acids and mood disorders}

\author{
C Owen ${ }^{1,2}$, A-M Rees ${ }^{1}$, G Parker ${ }^{1}$ \\ 'The Black Dog Institute, Sydney, Australia; and 2University of New South Wales
}

Background: Over the past century, consumption of omega-3 polyunsaturated fatty acids has decreased dramatically in Western diets, while consumption of omega- 6 has increased. There is mounting evidence suggesting that deficits in dietary intake of omega-3 are associated with a range of health problems, including mood disorders. This review will outline the epidemiological and clinical evidence for an association between omega-3 depletion and mood disorders, including unipolar, bipolar and perinatal depression and suicide risk.

Method: Relevant published studies are reviewed and theories regarding the mechanisms of action are presented.

Results: Strong correlations between rates of seafood consumption and mood disorders have been observed in several epidemiological studies. Biochemical studies indicate that omega-3 levels are lower in the tissue of people with depressive disorders than matched controls. The results of several randomized controlled trials indicate that omega-3 supplementation may have therapeutic benefit for depression suffers, although not all trials have shown a benefit.

Conclusions: Deficits in omega- 3 fatty acids are associated with increased prevalence of mood disorders and offer a potential rational treatment approach. A similar relationship between omega-3 depletion and coronary artery disease may explain the well-described links between coronary artery disease and depression. Inconsistency in clinical trial results to date may reflect differences in the type and dose of omega-3 supplement chosen, the depression subtype and/or baseline omega-3 levels of the study participants.

\section{Meanings of religion and spirituality for adolescent out-patients}

\section{T Perich', M Dudley², DH Pavlovic' ${ }^{1}$, F Fitzjames ${ }^{2}$ \\ 'The Black Dog Institute, Sydney, Australia; and 2Prince of Wales Hospital,} Randwick, Australia

Spirituality is an important but often overlooked area in mental health. This study aimed to examine individual meanings regarding the concepts of religion and spirituality for adolescents attending a mental health out-patient service. Participants were 53 adolescent out-patients of the Department of Child and Adolescent Mental Health, Prince of Wales Hospital, Sydney, 